In the first paper, actual uniformity field trials are examined and it is found that analyses based on conventional mathematical models may assess very poorly the probabilities used in detecting significantly different varieties.

Monte Carlo results show changes in the mathematical model of field trials that can give probability distributions that correspond closely to the distributions observed for actual trials.

In the second paper, emphasis is placed on reproducibility of field plot results as the most desirable evaluation. Techniques by which a stable ranking among treatments can be obtained (i.e.: $A$ is better than B) are discussed as a matter of field plot manipulation. Examples are given where reproducibility, as measured by the SD technique in a single year, is applicable to a high degree of certainty to results based on several years' experience. The SD technique provides a confidence limit depending on design, and the values of the limits are computed.

A reproducible ranking order is held to be desirable and the problems of securing one are discussed. Techniques are offered which simplify obtaining a stable ranking. Mathematical formulas are given by which given cut-off points of confidence can be calculated. Adequate field plot decisions are based on both agronomic usefulness and mathematical confidence. The SD technique is shown to fulfill both of these considerations.

\section{THE AUTHORS:}

George A. Baker is Professor of Mathematics and Statistician in the Experiment Station, Davis; John P. Johnson was at the time of these studies graduate student at the University of California, Davis, and is at present graduate student in statistics at Iowa State University, Ames; Burton J. Hoyle is Specialist in Field Station Administration and Superintendent of the Tulelake Field Station, Tulelake. 


\title{
Uniformity Field Trials and Monte Carlo Simulations $^{1,2}$
}

\begin{abstract}
INTRODUCTION
Examination of actual uniformity field trials shows that the ordinary analyses of variance of such trials give erratic results both from the standpoint of errors of the first kind and errors of the second kind. An error of the first kind is made when we say that a difference between varieties exists when, in fact, there is no difference. An error of the second kind is made when we say that no difference exists when, in fact, there is a difference. Monte Carlo simulations of uniformity trials, theoretical models, and detailed scrutiny of real trials indicate that island-like fertility levels with random elements whose variabilities depend upon the fertility levels are realistic and greatly disturb the validity of the conventional analyses.

Concern over the adequacy of the conventional model for the analyses of field trials has been expressed for a long

time (Baker, 1941). Attempts to clarify the difficulties have been made by Baker (1952) and Baker and various coauthors (1944, 1949, 1950, 1952, 1953, $1957,1961)$. In general, it has been found that errors of the first kind may be over- or underestimated and that the same is true for errors of the second kind. There is a distinct tendency for fertility levels to occur in the form of islands that cannot be assessed well before the results are observed. The variability of the observed responses depends on the fertility level.

This paper presents further Monte Carlo results concerning possible disturbing elements present in actual trials that are not realistically allowed for in the presently used mathematical models. Also, a brief indication of the possibility of more realistic mathematical models is given.
\end{abstract}

\section{ACTUAL FIELD TRIALS}

Table 1 concerns errors of the first kind. In this table, we have taken the 57 tenth-acre barley plots in the uniformity trial reported by Baker, et al. (1952) and regarded them as three randomized blocks of 19 varieties for seven of the years. One hundred random assignments of varieties were made. The mean yields and standard deviations of
57 plots are given. The mean of the $100 \mathrm{~F}$ 's and their variances are listed along with the number of values exceeding the values of $F$ for the 5 and 1 per cent levels indicated by the conventional normal model. The means and variances of correlations between observed values and residuals based on 20 trials (except 8 trials for 1925) are given also.

\footnotetext{
${ }^{1}$ Submitted for publication April 15, 1964.

${ }^{2}$ Part of the computing for this project was done by the Computer Center, University of California, Davis. The Computer Center is partially supported by National Institute of Health Grant No. FR-00009.
} 
TABle 1

ERRORS OF THE FIRST KIND*

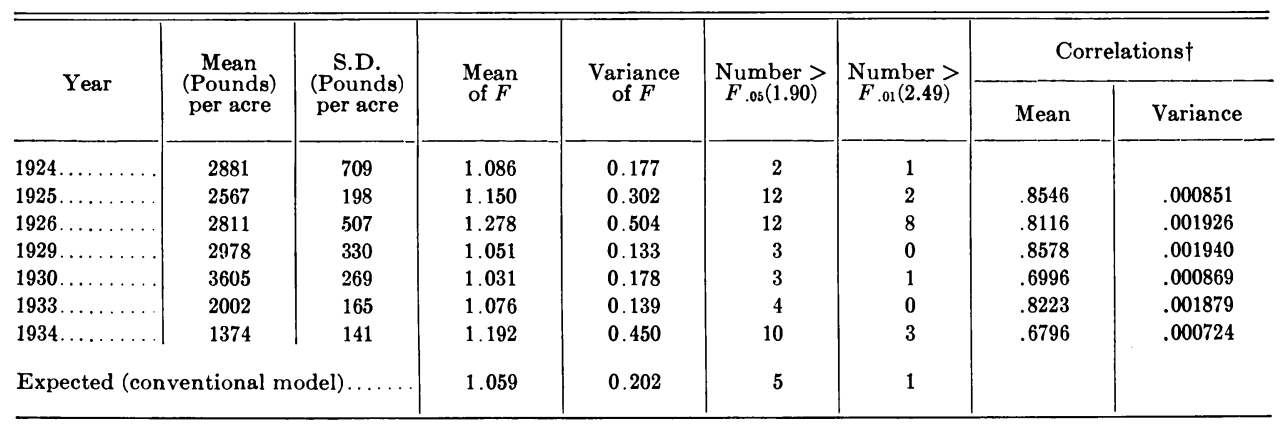

* Means and variances for $100 F$-values for 57 barley plots regarded as three randomized blocks of 19 varieties for seven of the years as given in Baker et al. (1952) along with the number of values exceeding 5 and 1 per cent levels. Means and variances of correlations between observed values and residuals are based on 20 tr_als, except in 1925 when they were based on eight trials.

$\dagger$ Professor P. W. M. John of the Mathematics Department, University of California, Davis, has pointed out that the square of the correlation coefficients in tables $1,3,4$, and 5 can be computed as the ratio of the error sum of squares to the total sum of squares in the corresponding analysis of variance tables.

This table indicates considerable variation in the year-to-year behavior of the conventional $\boldsymbol{F}$-test for significance of varieties as far as errors of the first kind ( $\alpha$-errors) are concerned.

Table 2 considers the behavior of some of the years listed in table 1 with respect to errors of the second kind ( $\beta$-errors). Real differences were applied to varieties for the years 1925 , 1930,1934 . The magnitude of the real differences is indicated by the value of Tang's $\varphi$. For a complete discussion of $\varphi$ see Baker and Roessler (1957).

The years 1925 and 1934 behaved as expected under the conventional model, but in 1930, significantly fewer errors of the second kind were found than expected.

Results for 25 actual $9 \times 9$ latin squares are listed in table 4 . These results for the $a$-error situation seem to be different from the normal conventional model as given in its first line.

TABLE 2

ERRORS OF THE SECOND KIND*

\begin{tabular}{c|c|c|c|c|c|c|c|c|c}
\hline Year & Mean & SD & $\begin{array}{c}\text { Number } \\
\text { of } F \text { 's }\end{array}$ & $\varphi$ & $a$ & $\beta$ & $\begin{array}{c}\text { Number }> \\
F .05\end{array}$ & $\begin{array}{c}\text { Number } \\
F .01\end{array}$ & $\chi^{2}-1 \mathrm{df}$ \\
\hline $1925 \ldots$ & 2568 & 194 & 100 & 1.44 & 0.01 & 0.8 & 95 & 82 & 0.25 \\
$1930 \ldots$ & 3604 & 273 & 100 & 1.27 & 0.01 & 0.7 & 80 & 54 & 12.18 \\
$1934 \ldots$ & 1374 & 143 & 50 & 2.32 & 0.01 & $>0.8$ & 50 & 50 & $\ldots$. \\
\hline
\end{tabular}

* Real differences were applied to varieties in the set-up for the previous table. The values of Tang's $\varphi$ (see Baker et al., 1952) are given.

\section{EFFECT OF UNIFORM FUNDAMENTAL ERROR DISTRIBUTIONS ON a-ERRORS}

Since the actual data considered in the previous section indicate considerable possible deviation of field trials from the common normal-error distribution model, it becomes of interest to pinpoint the cause of failure, if possible. To examine the possible effect of distor- tions of the fundamental error distribution, we considered an extensive set of quite different distributions by Monte Carlo methods for $6 \times 6,9 \times 9$ and $12 \times$ 12 latin squares. These results are given in tables 3,4 and 5. The populations I, II, III, IV and $\mathrm{V}$ are given in detail by 
TABle 3

MEANS AND VARIANCES OF VARIETY $F$-VALUES AND CORRELATIONS BETWEEN OBSERVED AND RESIDUAL VALUES FOR SIMULATED UNIFORMITY $6 \times 6$ LATIN SQUARES

\begin{tabular}{|c|c|c|c|c|c|}
\hline \multirow{2}{*}{ Population } & \multirow{2}{*}{ Number } & \multicolumn{2}{|c|}{$F$-values } & \multicolumn{2}{|c|}{ Correlations } \\
\hline & & Mean & Variance & Mean & Variance \\
\hline Normal........... & 100 & 1.0888 & .627015 & .7596 & .005058 \\
\hline I........ & 100 & 1.1988 & 1.754286 & .7377 & .007527 \\
\hline II. . & 100 & 1.0548 & .541458 & .7564 & .006185 \\
\hline III.... & 100 & 1.2586 & 1.276479 & .7363 & .007698 \\
\hline IV .... & 100 & 1.1070 & .550421 & .7558 & .003907 \\
\hline$V \ldots \ldots$ & 100 & 1.0124 & .651804 & .7620 & .006446 \\
\hline
\end{tabular}

TABLE 4

MEANS AND VARIANCES OF VARIETY $F$-VALUES AND CORRELATIONS BETWEEN OBSERVED AND RESIDUAL VALUES FOR SIMULATED AND ACTUAL UNIFORMITY $9 \times 9$ LATIN SQUARE TRIALS

\begin{tabular}{|c|c|c|c|c|c|}
\hline Population & Number & Mean & Variance & Mean & Variance \\
\hline Normal. & 100 & 1.0050 & .424621 & .8424 & .001562 \\
\hline I......... & 100 & 1.0121 & .263890 & .8414 & .001161 \\
\hline II $\ldots \ldots$ & 100 & 1.0337 & .260448 & .8360 & .001755 \\
\hline IV ...... & 100 & 0.9880 & .248998 & .8483 & .001498 \\
\hline $\mathbf{V} \ldots \ldots \ldots$ & 100 & 1.0061 & .257487 & .8414 & .001669 \\
\hline $2 N(15.5,5)+1 N(75.5,5)$ & 50 & 0.9876 & .289900 & .8350 & .002293 \\
\hline$N(15.5,5)+N(65.5,5)$ & 100 & 1.0761 & .426100 & .8376 & .002344 \\
\hline$N(15.5,5)+N(60.0,20)$ & 100 & 0.5255 & .061327 & .9211 & .000308 \\
\hline Sum of rectangular and normal. . & 100 & 1.0255 & .014394 & .8414 & .001128 \\
\hline
\end{tabular}

* These data were furnished by B. J. Hoyle, Superintendent of the University of California Tulelake Field Station.

TABLE 5

MEANS AND VARIANCES OF VARIETY $F$-VALUES AND CORRELATIONS BETWEEN OBSERVED AND RESIDUAL VALUES FOR SIMULATED UNIFORMITY $12 \times 12$ LATIN SQUARE TRIALS

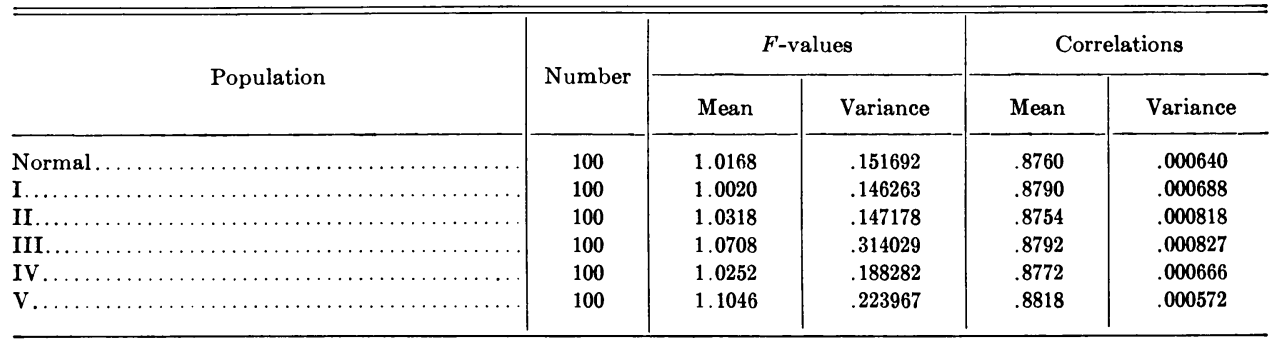

Baker (1958). These populations are composed of two normal populations and are distinctly non-normal.

Considerable disturbance of the F- distributions was achieved by manipulation of the error distributions, but perhaps not enough to account for the observed failures for actual field trials. 


\section{EFFECT OF DIFFERENCES IN FERTILITY LEVELS AND ERROR VARIANCES OF SUBPLOTS}

Uniformity field trials, when differences in fertility levels of subplots are not included in experimental error for two randomized blocks with two subplots each, have been discussed by Baker (1952). Selected ordinates for systematic and randomized procedures for seven pairs of values of the parameters $m_{1} / \sigma$ and $m_{2} / \sigma$ for the corresponding $F$-distributions are given in table 1 of this paper. These parameters measure the inequality of the fertility levels of

TABLE 6

SELECTED ORDINATES OF $\boldsymbol{F}$ DISTRIBUTIONS CORRESPONDING TO $m_{1}=m_{2}=0$

AND VARIOUS VALUES OF $r$

\begin{tabular}{|c|c|c|c|}
\hline$F / r$ & 0.0 & .5 & .9 \\
\hline (3) $1 \ldots$ & 31.828 & 27.566 & 13.878 \\
\hline (2)5 . . & 4.479 & 3.898 & 1.984 \\
\hline $.01 \ldots$ & 3.152 & 2.756 & 1.419 \\
\hline $.025 \ldots$ & 1.964 & 1.742 & .928 \\
\hline $.05 \ldots \ldots$ & 1.356 & 1.230 & .693 \\
\hline $.1 \ldots \ldots$ & .915 & .864 & .545 \\
\hline $.2 \ldots$ & .593 & .597 & .470 \\
\hline $.4 \ldots \ldots$ & .359 & .391 & .463 \\
\hline 6 & .257 & .291 & .465 \\
\hline $.8 \ldots$ & .198 & .227 & .431 \\
\hline $1.0 \ldots$ & .159 & .184 & .365 \\
\hline $1.2 \ldots$ & .132 & .152 & .293 \\
\hline $1.4 \ldots \ldots$ & .112 & .128 & .230 \\
\hline $1.6 \ldots$ & .097 & .110 & .181 \\
\hline $1.8 \ldots \ldots$ & .085 & .095 & .144 \\
\hline 2.0 . & .075 & .084 & .117 \\
\hline 2.2 . & .067 & .074 & .096 \\
\hline $2.4 \ldots \ldots$ & .060 & .066 & .080 \\
\hline $2.6 \ldots$ & .055 & .059 & .068 \\
\hline $2.8 \ldots \ldots$ & .050 & .054 & .059 \\
\hline $3.0 \ldots \ldots$ & .046 & .049 & .051 \\
\hline $3.2 \ldots$ & .042 & .045 & .045 \\
\hline $3.4 \ldots \ldots$ & .039 & .041 & .040 \\
\hline $3.6 \ldots \ldots$ & .036 & .038 & .035 \\
\hline $3.8 \ldots \ldots$ & .034 & .035 & .032 \\
\hline $4.0 \ldots \ldots$ & .032 & .033 & .029 \\
\hline $4.2 \ldots \ldots$ & .030 & .031 & .026 \\
\hline $4.4 \ldots \ldots$ & .028 & .029 & .024 \\
\hline $4.6 \ldots \ldots$ & .026 & .027 & .022 \\
\hline $4.8 \ldots \ldots$ & .025 & .025 & .020 \\
\hline $5.0 \ldots \ldots$ & .024 & .024 & .019 \\
\hline $100.0 \ldots$ & (3)31 & (3) 28 & (3) 14 \\
\hline $10,000.0 \ldots \ldots$ & (6)32 & (6) 28 & (6) 14 \\
\hline
\end{tabular}

the subplots in terms of the uniform standard deviation of the experiment. It was seen that the tails of some of the $F$-distributions are heavier than for the conventional model, indicating that much larger values of $F$ are required for significance. On the other hand, some of the tails were lighter, so that smaller $F$-values are indicative of significance at the usual levels. Randomization is effective in some cases in giving a distribution that is closer to the con-

TABLE 7

SELECTED ORDINATES OF $F$ DISTRIBUTIONS CORRESPONDING TO $m_{1}=0, m_{2} / \sigma=1$, AND VARIOUS VALUES OF $r$

\begin{tabular}{|c|c|c|c|}
\hline$F / r$ & 0.0 & .5 & .9 \\
\hline (3) $1 \ldots$ & 19.306 & 19.435 & 21.933 \\
\hline (2)5 . & 2.730 & 2.740 & 3.093 \\
\hline $.01 \ldots$ & 1.930 & 1.932 & 2.181 \\
\hline $.025 \ldots$ & 1.220 & 1.211 & 1.367 \\
\hline $.05 \ldots$ & .862 & . .845 & .949 \\
\hline .1 & .607 & .583 & .642 \\
\hline $.2 \ldots \ldots$ & .423 & .397 & .401 \\
\hline $.4 \ldots \ldots$ & .287 & .267 & .201 \\
\hline $6 \ldots \ldots$ & .222 & .211 & .117 \\
\hline $.8 \ldots \ldots$ & .182 & .178 & .085 \\
\hline 1.0. & .154 & .154 & .077 \\
\hline $1.2 \ldots \ldots$ & .133 & .136 & .082 \\
\hline $1.4 \ldots \ldots$ & .116 & .121 & .090 \\
\hline 1.6. & .103 & .109 & .098 \\
\hline $1.8 \ldots \ldots$ & .093 & .098 & .102 \\
\hline 2.0 . & .084 & .089 & .103 \\
\hline 2.2. & .076 & .082 & .101 \\
\hline $2.4 \ldots \ldots$ & .070 & .075 & .097 \\
\hline $2.6 \ldots \ldots$ & .064 & .069 & .092 \\
\hline $2.8 \ldots \ldots$ & .059 & .064 & .087 \\
\hline 3.0 . & .055 & .059 & .081 \\
\hline $3.2 \ldots$ & .051 & .055 & .076 \\
\hline $3.4 \ldots \ldots$ & .048 & .051 & .071 \\
\hline $3.6 \ldots \ldots$ & .045 & .048 & .066 \\
\hline $3.8 \ldots \ldots$ & .042 & .045 & .061 \\
\hline 4.0. & .039 & .042 & .057 \\
\hline $4.2 \ldots \ldots$ & .037 & .040 & .053 \\
\hline $4.4 \ldots \ldots$ & .035 & .038 & .050 \\
\hline $4.6 \ldots \ldots$ & .033 & .036 & .047 \\
\hline $4.8 \ldots \ldots$ & .032 & .034 & .044 \\
\hline $5.0 \ldots \ldots$ & .030 & .032 & .042 \\
\hline $100.0 \ldots \ldots$ & (3) 46 & (3) 44 & (3) 41 \\
\hline $10,000.0 \ldots \ldots$ & (6) 47 & (6) 44 & (6) 40 \\
\hline
\end{tabular}


TABLE 8

TABLE 9

SELECTED ORDINATES OF $F$ DISTRI- SELECTED ORDINATES OF $F$ DISTRIBUTIONS CORRESPONDING TO

$m_{1} / \sigma=1, m_{2}=0$,

AND VARIOUS VALUES OF $r$

\begin{tabular}{|c|c|c|c|}
\hline$F / r$ & 0.0 & .5 & .9 \\
\hline (3) $1 \ldots$ & 46.516 & 44.125 & 40.030 \\
\hline . (2)5 5 & 6.521 & 6.217 & 5.706 \\
\hline .01 & 4.570 & 4.379 & 4.067 \\
\hline $.025 \ldots$ & 2.816 & 2.736 & 2.631 \\
\hline .05 & 1.908 & 1.893 & 1.924 \\
\hline $.1 \ldots \ldots$ & 1.244 & 1.275 & 1.432 \\
\hline $.2 \ldots \ldots$ & .758 & .807 & 1.048 \\
\hline $.4 \ldots \ldots$ & .417 & .450 & .591 \\
\hline $.6 \ldots \ldots$ & .276 & .292 & .278 \\
\hline .8 & .200 & .206 & .131 \\
\hline $1.0 \ldots \ldots$ & .154 & .154 & .077 \\
\hline $1.2 \ldots$ & .123 & .120 & .057 \\
\hline $1.4 \ldots$ & .101 & .097 & .048 \\
\hline $1.6 \ldots \ldots$ & .084 & .081 & .043 \\
\hline $1.8 \ldots \ldots$ & .072 & .068 & .040 \\
\hline 2.0 & .062 & .059 & .037 \\
\hline $2.2 \ldots$ & .055 & .051 & .035 \\
\hline $2.4 \ldots \ldots$ & .049 & .045 & .033 \\
\hline 2.6 & .043 & .040 & .031 \\
\hline 2.8 & .039 & .036 & .029 \\
\hline $3.0 \ldots$ & .035 & .033 & .027 \\
\hline 3.2 . & .032 & .030 & .026 \\
\hline $3.4 \ldots$ & .029 & .027 & .025 \\
\hline $3.6 \ldots$ & .027 & .025 & .023 \\
\hline 3.8. & .025 & .023 & .022 \\
\hline 4.0 . & .023 & .022 & .021 \\
\hline $4.2 \ldots$ & .021 & .020 & .020 \\
\hline $4.4 \ldots \ldots$ & .020 & .019 & .019 \\
\hline $4.6 \ldots \ldots$ & .019 & .018 & .018 \\
\hline $4.8 \ldots$ & .018 & .017 & .017 \\
\hline $5.0 \ldots \ldots$ & .017 & .016 & .016 \\
\hline $100.0 \ldots$ & (3) 19 & (3)19 & (3) 22 \\
\hline $10,000.0 \ldots$ &.$(6) 19$ & (6) 19 & .(6) 22 \\
\hline
\end{tabular}

BUTIONS CORRESPONDING TO

RANDOMIZATION OF THE

PARAMETERS VALUES OF TABLES

7 AND 8 FOR VARIOUS VALJUES OF $r$

\begin{tabular}{|c|c|c|c|}
\hline$F / r$ & 0.0 & .5 & .9 \\
\hline (3) 1 . & 32.911 & 31.780 & 30.982 \\
\hline . (2) $5 \ldots$ & 4.626 & 4.478 & 4.400 \\
\hline $.01 \ldots$ & 3.250 & 3.156 & 3.124 \\
\hline .025 & 2.018 & 1.974 & 1.999 \\
\hline .05 & 1.385 & 1.369 & 1.436 \\
\hline $.1 \ldots$ & .926 & .929 & 1.032 \\
\hline .2 . & .590 & .602 & .724 \\
\hline $.4 \ldots \ldots$ & .352 & .358 & .396 \\
\hline $.6 \ldots$ & .249 & .252 & .198 \\
\hline .8 & .191 & .192 & .108 \\
\hline $1.0 \ldots$ & .154 & .154 & .077 \\
\hline 1.2. & .128 & .128 & .070 \\
\hline $1.4 \ldots$ & .108 & .109 & .069 \\
\hline $1.6 \ldots \ldots$ & .094 & .095 & .070 \\
\hline $1.8 \ldots$ & .082 & .083 & .071 \\
\hline 2.0 . & .073 & .074 & .070 \\
\hline $2.2 \ldots$ & .066 & .066 & .068 \\
\hline $2.4 \ldots \ldots$ & .060 & .060 & .065 \\
\hline $2.6 \ldots$ & .054 & .054 & .062 \\
\hline $2.8 \ldots$ & .049 & .050 & .058 \\
\hline $3.0 \ldots$ & .045 & .046 & .054 \\
\hline $3.2 \ldots$ & .041 & .042 & .051 \\
\hline $3.4 \ldots \ldots$ & .038 & .039 & .048 \\
\hline $3.6 \ldots$ & .035 & .036 & .045 \\
\hline $3.8 \ldots$ & .033 & .034 & .042 \\
\hline $4.0 \ldots$ & .031 & .032 & .039 \\
\hline $4.2 \ldots$ & .029 & .030 & .036 \\
\hline $4.4 \ldots$ & .027 & .028 & .034 \\
\hline 4.6. & .026 & .027 & .032 \\
\hline 4.8 & .025 & .026 & .030 \\
\hline 5.0 . & .024 & .024 & .029 \\
\hline $100.0 \ldots$ & (3)32 & (3)32 & .(3) 32 \\
\hline $10,000.0 \ldots$ & . (6)33 & (6)32 & (6)31 \\
\hline
\end{tabular}


TABLE 10

SELECTED ORDINATES OF $F$ DISTRIBUTIONS CORRESPONDING TO $m_{1} / \sigma=1, m_{2} / \sigma=1$ AND VARIOUS VALUES OF $r$

\begin{tabular}{|c|c|c|c|c|c|}
\hline$F / r$ & -.9 & -.5 & 0.0 & .5 & .9 \\
\hline (3) $1 \ldots \ldots \ldots$ & 45.958 & 37.012 & 28.218 & 19.441 & 8.638 \\
\hline $.01 \ldots \ldots \ldots \ldots \ldots \ldots$ & 4.461 & 3.623 & 2.821 & 1.984 & .890 \\
\hline $.025 \ldots \ldots \ldots \ldots \ldots \ldots$ & 2.693 & 2.220 & 1.783 & 1.293 & .588 \\
\hline $.05 \ldots \ldots \ldots \ldots \ldots \ldots$ & 1.757 & 1.493 & 1.257 & .957 & .448 \\
\hline $.1 \ldots \ldots \ldots \ldots \ldots \ldots$ & 1.050 & .962 & .879 & .732 & .367 \\
\hline $.2 \ldots \ldots$ & .533 & .580 & .600 & .580 & .349 \\
\hline $.4 \ldots \ldots \ldots \ldots \ldots$ & .246 & .323 & .385 & .454 & .433 \\
\hline $.6 \ldots \ldots \ldots$ & .167 & .222 & .282 & .368 & .548 \\
\hline 8 & .127 & .168 & .220 & .299 & .592 \\
\hline $1.8 \ldots \ldots \ldots$ & .055 & .074 & .093 & .119 & .162 \\
\hline $2.0 \ldots$ & .049 & .066 & .082 & .102 & .123 \\
\hline $2.2 \ldots \ldots$ & .045 & .059 & .073 & .088 & .096 \\
\hline $2.4 \ldots \ldots$ & .041 & .054 & .065 & .077 & .077 \\
\hline $2.6 \ldots \ldots \ldots \ldots$ & .038 & .049 & .059 & .068 & .063 \\
\hline $2.8 \ldots \ldots \ldots \ldots$ & .035 & .045 & .053 & .061 & .052 \\
\hline $3.0 \ldots$ & .033 & .042 & .049 & .054 & .044 \\
\hline $3.2 \ldots$ & .031 & .039 & .045 & .049 & .038 \\
\hline $3.4 \ldots \ldots \ldots \ldots$ & .029 & .036 & .041 & .044 & .033 \\
\hline $4.8 \ldots \ldots \ldots \ldots$ & .022 & .024 & .025 & .025 & .015 \\
\hline $5.0 \ldots \ldots \ldots \ldots \ldots \ldots$ & .021 & .023 & .024 & .023 & .014 \\
\hline $100.0 \ldots \ldots \ldots \ldots \ldots \ldots$ & (3) 45 & .(3) 36 & (3) 28 & (3) 20 & (4) 89 \\
\hline $10,000.0 \ldots \ldots \ldots \ldots \ldots \ldots$ & .(6) 46 & (6) 37 & (6) 28 & (6) 19 &.$(7) 86$ \\
\hline
\end{tabular}


TABLE 11

SELECTED ORDINATES OF $F$ DISTRIBUTIONS CORRESPONDING TO $m_{1}=0, m_{2} / \sigma=2$, AND VARIOUS VALUES OF $r$

\begin{tabular}{|c|c|c|c|}
\hline$F / r$ & 0.0 & .5 & .9 \\
\hline (3) $1 \ldots$ & 4.309 & 5.973 & 9.715 \\
\hline (2)5 $\ldots$ & .618 & .848 & 1.360 \\
\hline $.01 \ldots$. & .444 & .602 & .952 \\
\hline $.025 \ldots$ & .293 & .385 & .584 \\
\hline $.05 \ldots$. & .221 & .277 & .394 \\
\hline $.1 \ldots \ldots$ & .175 & .202 & .259 \\
\hline $.2 \ldots \ldots$ & .147 & .150 & .166 \\
\hline $.4 \ldots \ldots$ & .131 & .116 & .109 \\
\hline $.6 \ldots \ldots$ & .123 & .102 & .085 \\
\hline $.8 \ldots \ldots$ & .116 & .094 & .071 \\
\hline $1.0 \ldots \ldots$ & .109 & .090 & .060 \\
\hline $1.2 \ldots \ldots$ & .103 & .086 & .053 \\
\hline $1.4 \ldots \ldots$ & .097 & .083 & .047 \\
\hline $1.6 \ldots \ldots$ & .091 & .080 & .043 \\
\hline $1.8 \ldots \ldots$ & .086 & .077 & .041 \\
\hline $2.0 \ldots \ldots$ & .081 & .074 & .040 \\
\hline $2.2 \ldots \ldots$ & .076 & .072 & .041 \\
\hline $2.4 \ldots \ldots$ & .072 & .069 & .043 \\
\hline $2.6 \ldots \ldots$ & .068 & .067 & .045 \\
\hline $2.8 \ldots \ldots$ & .065 & .064 & .047 \\
\hline $3.0 \ldots \ldots$ & .062 & .061 & .048 \\
\hline $3.2 \ldots \ldots$ & .059 & .059 & .049 \\
\hline $3.4 \ldots \ldots$ & .056 & .057 & .050 \\
\hline $3.6 \ldots \ldots$ & .053 & .054 & .050 \\
\hline $3.8 \ldots \ldots$ & .051 & .052 & .050 \\
\hline $4.0 \ldots \ldots$ & .049 & .050 & .050 \\
\hline $4.2 \ldots \ldots$ & .047 & .048 & .049 \\
\hline $4.4 \ldots \ldots$ & .045 & .046 & .049 \\
\hline $4.6 \ldots \ldots$ & .043 & .044 & .048 \\
\hline $4.8 \ldots \ldots$ & .041 & .042 & .047 \\
\hline $5.0 \ldots \ldots$ & .039 & .041 & .046 \\
\hline $100.0 \ldots$ & .(3)78 & .(3)78 & (3) 80 \\
\hline $10,000.0 \ldots \ldots$ & .(6) 80 & .(6) 80 & (6) 80 \\
\hline
\end{tabular}

TABLE 12

SELECTED ORDINATES OF $F$ DISTRIBUTIONS FOR $m_{1} / \sigma=2, m_{2}=0$, AND VARIOUS VALUES OF $r$

\begin{tabular}{|c|c|c|c|}
\hline$F / r$ & 0.0 & .5 & .9 \\
\hline .(3) $1 \ldots$ & 80.468 & 80.014 & 79.787 \\
\hline (2) $5 \ldots$ & 11.189 & 11.184 & 11.288 \\
\hline $.01 \ldots \ldots$ & 7.777 & 7.814 & 7.980 \\
\hline $.025 \ldots$ & 4.676 & 4.761 & 5.026 \\
\hline $.05 \ldots$ & 3.046 & 3.156 & 3.486 \\
\hline $.1 \ldots \ldots$ & 1.845 & 1.947 & 2.268 \\
\hline $.2 \ldots$ & .987 & 1.035 & 1.141 \\
\hline $.4 \ldots \ldots$ & .440 & .424 & .274 \\
\hline $.6 \ldots$ & .248 & .220 & .118 \\
\hline $.8 \ldots \ldots$ & .158 & .113 & .080 \\
\hline $1.0 \ldots \ldots$ & .109 & .090 & .060 \\
\hline $1.2 \ldots \ldots$ & .080 & .065 & .048 \\
\hline $1.4 \ldots \ldots$ & .061 & .049 & .039 \\
\hline $1.6 \ldots \ldots$ & .048 & .039 & .033 \\
\hline $1.8 \ldots \ldots$ & .038 & .032 & .028 \\
\hline 2.0 . & .032 & .027 & .024 \\
\hline $2.2 \ldots \ldots$ & .027 & .023 & .021 \\
\hline $2.4 \ldots \ldots$ & .023 & .020 & .019 \\
\hline $2.6 \ldots \ldots$ & .020 & .017 & .017 \\
\hline $2.8 \ldots \ldots$ & .017 & .015 & .015 \\
\hline $3.0 \ldots \ldots$ & .015 & .013 & .013 \\
\hline $3.2 \ldots \ldots$ & .013 & .012 & .012 \\
\hline $3.4 \ldots \ldots$ & .012 & .011 & .011 \\
\hline $3.6 \ldots \ldots$ & .011 & .010 & .010 \\
\hline $3.8 \ldots \ldots$ &.$(2) 97$ & (2)93 & .(2) 97 \\
\hline $4.0 \ldots \ldots$ & (2)88 & (2)86 & (2) 91 \\
\hline $4.2 \ldots$ &.$(2) 81$ & .(2)79 & (2) 85 \\
\hline $4.4 \ldots \ldots$ &.$(2) 74$ & .(2)74 & (2) 79 \\
\hline $4.6 \ldots \ldots$ &.$(2) 69$ & .(2) 69 & .(2) 74 \\
\hline $4.8 \ldots \ldots$ &.$(2) 63$ &.$(2) 64$ & .(2)70 \\
\hline $5.0 \ldots \ldots$ & .(2)59 & .(2)60 & .(2) 66 \\
\hline $100.0 \ldots \ldots$ &.$(4) 44$ &.$(4) 60$ & .(4) 95 \\
\hline $10,000.0 \ldots \ldots$ &.$(7) 43$ &.$(7) 60$ & .(7) 97 \\
\hline
\end{tabular}


TABLE 13

SELECTEI) ORDINATES OF $F$ DISTRIBUTIONS FOR RANIOMIZATION FOR THE VALUES OF THE PARAMETERS F()R TABLES 11 ANI) 12, AND VARIOUS VALUES OF $r$

\begin{tabular}{|c|c|c|c|}
\hline$F / r$ & 0.0 & .5 & .9 \\
\hline (3) $1 \ldots$ & 42.388 & 42.994 & 44.751 \\
\hline (2)5 5 & 5.904 & 6.016 & 6.324 \\
\hline $01 \ldots$ & 4.110 & 4208 & 4.466 \\
\hline .025 & 2.484 & 2.573 & 2.805 \\
\hline $.05 \ldots \ldots$ & 1.634 & 1.716 & 1.940 \\
\hline .1 & 1.010 & 1.074 & 1.264 \\
\hline .2 & .567 & .592 & .654 \\
\hline $4 \ldots \ldots$ & .286 & .270 & .192 \\
\hline .6 & .186 & .161 & .102 \\
\hline $.8 \ldots \ldots$ & .137 & .104 & .076 \\
\hline $1.0 \ldots \ldots$ & .109 & .090 & .060 \\
\hline $1.2 \ldots \ldots$ & .092 & .076 & .050 \\
\hline $1.4 \ldots \ldots$ & .079 & .066 & .043 \\
\hline $1.6 \ldots$ & .070 & .060 & .038 \\
\hline $1.8 \ldots \ldots$ & .062 & .054 & .034 \\
\hline 2.0 . & .056 & .050 & .032 \\
\hline 2.2 & .052 & .047 & .031 \\
\hline $2.4 \ldots \ldots$ & .048 & .044 & .031 \\
\hline 2.6 . & .044 & .042 & .031 \\
\hline 2.8 & .041 & .040 & .031 \\
\hline 3.0 . & .038 & .037 & .030 \\
\hline $3.2 \ldots \ldots$ & .036 & .035 & .030 \\
\hline $3.4 \ldots \ldots$ & .034 & .034 & .030 \\
\hline 3.6 . & .032 & .032 & .030 \\
\hline 3.8 . & .030 & .030 & .030 \\
\hline 4.0 . & .028 & .029 & .029 \\
\hline $4.2 \ldots \ldots$ & .027 & .028 & .029 \\
\hline $4.4 \ldots \ldots$ & .026 & .027 & .028 \\
\hline $4.6 \ldots \ldots$ & .025 & .025 & .028 \\
\hline $4.8 \ldots \ldots$ & .024 & .024 & .027 \\
\hline $5.0 \ldots \ldots$ & .023 & .023 & .026 \\
\hline $100.0 \ldots \ldots$ & (3) 41 & (3) 42 & .(3) 45 \\
\hline $10,000.0 \ldots \ldots$ & (6) 42 & (6) 43 & .(6) 45 \\
\hline
\end{tabular}

ventional $F$-distribution than is the $F$ distribution for a systematic procedure.

In the present study, we permit the variances of the yields in the subplot to vary as well as the fertility levels within the subplots. We express the extent of the differences between the variances by means of a parameter $r$. Tables 6,7 , $8,9,10,11,12,13$ and 14 give the $F$-distributions corresponding to 9 columns of table 1, Baker (1952), for $r=-0.9$, $-0.5,0.0,0.5,0.9$. The $F$-distributions for $r=0.0$ in these tables are the same as for table 1 in the previous publication except for computing errors, mainly for columns 5 and 9 . All of these tables except 10 and 14 are symmetrical with respect to plus and minus values of $r$ and hence the columns for negative $r$ are omitted.

It is seen that far greater distortions in the $\boldsymbol{F}$-distributions are possible with the present model than with the previous one (Baker, 1952).

The details of the development of the present model are as follows.

As in Baker (1952), two randomized blocks with two subplots each shall be considered, and in place of the standard mathematical model,

$$
\begin{aligned}
& v_{i j}=g+b_{i}+t_{j}+\epsilon_{i j}, \\
& \quad i=1,2 \text { and } j=1,2,
\end{aligned}
$$

where the random parts, $\boldsymbol{\epsilon}_{i j}$ 's, are assumed to be distributed independently as $N(o, \sigma)$, we shall assume that $\xi_{i j}$ is the "true" unknown fertility level in the $j$ th subplot of the $i$ th block and that our mathematical model is

$$
v_{i j}=x_{i j}+\xi_{i j}
$$

where the $x_{i j}$ are independently distributed with zero means and variances proportional to $\xi_{i j}$.

If we apply the conventional analysis of variance, we obtain 
TABLE 14

SELECTED ORDINATES OF $F$ DISTRIBUTIONS CORRESPONDING TO $m_{1} / \sigma=2, m_{2} / \sigma=2$, AND VARIOUS VALUES OF $r$

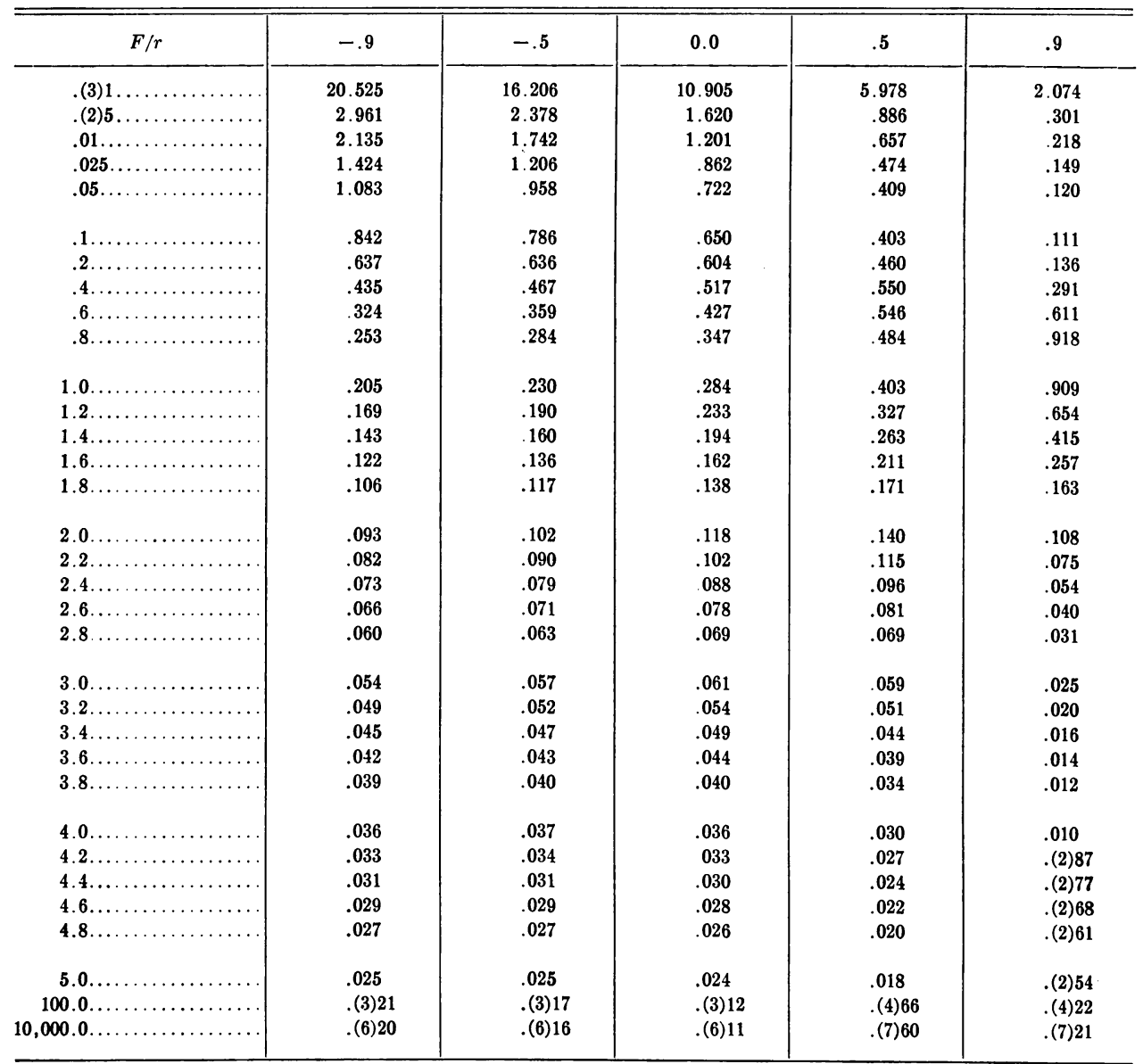

$$
S_{v}^{2}=\frac{1}{4}\left(v_{11}+v_{21}-v_{12}-v_{22}\right)^{2}
$$

and

$$
S_{e}^{2}=\frac{1}{4}\left(v_{11}-v_{21}-v_{12}+v_{22}\right)^{2}
$$

where $S_{v}^{2}$ is the variety sum of squares and $S_{e}^{2}$ is the error sum of squares each with one degree of freedom.

Put

$$
\begin{aligned}
& z_{1}=v_{11}+v_{21}-v_{12}-v_{22}, \\
& z_{2}=v_{11}-v_{21}-v_{12}+v_{22},
\end{aligned}
$$

and we get that $z_{1}$ and $z_{2}$ have a bivariate normal distribution with means

$$
\begin{aligned}
& m_{1}=\xi_{11}+\xi_{21}-\xi_{12}-\xi_{22} \\
& m_{2}=\xi_{11}-\xi_{21}-\xi_{12}+\xi_{22}
\end{aligned}
$$

and variances and covariance

$$
\begin{array}{r}
\operatorname{var}\left(z_{1}\right)=\operatorname{var}\left(z_{2}\right)=\sigma^{2}=\sigma_{11}^{2} \\
+\sigma_{21}^{2}+\sigma_{12}^{2}+\sigma_{22}^{2}
\end{array}
$$

$$
\operatorname{cov}\left(z_{1}, z_{2}\right)=r \sigma^{2}=\sigma_{11}^{2}-\sigma_{21}^{2}
$$

$$
+\sigma_{12}^{2}-\sigma_{22}^{2} \text {. }
$$

If we set $F=\left(z_{1} / z_{2}\right)^{2}$, then, by using equation (16), page 5, of Baker (1932), and transforming to a new variable, we have 


$$
\begin{gathered}
f\left(F, r, m_{1}, m_{2}, \sigma\right)=\frac{1}{2 \pi F^{1 / 2}}\left\{\left(\frac{1}{a_{1}}+\frac{1}{a_{2}}\right) \sqrt{1-r^{2}} \exp \left[-\frac{1}{2 \sigma^{2}}\left(m_{1}^{2}-2 r m_{1} m_{2}+m_{2}^{2}\right)\right]\right. \\
+\frac{b_{1}}{a_{1}^{3 / 2}} \exp \left[-\frac{1}{2 \sigma^{2} a_{1}}\left(m_{2}-F^{1 / 2} m_{1}\right)^{2}\right] \int_{0} b_{1}\left[a_{1}\left(1-r^{2}\right)\right]^{-1 / 2} e^{-u^{2} / 2} d u \\
\left.+\frac{b_{2}}{a_{2}^{3 / 2}} \exp \left[-\frac{1}{2 \sigma^{2} a_{2}}\left(m_{2}+F^{1 / 2} m_{1}\right)^{2}\right] \int_{0} b_{2}\left[a_{2}\left(1-r^{2}\right)\right]^{-1 / 2} e^{-u^{2} / 2} d u\right\}
\end{gathered}
$$

where

$$
\begin{aligned}
& a_{1}=1-2 r F^{1 / 2}+F \\
& a_{2}=1+2 r F^{1 / 2}+F \\
& b_{1}=\left(r m_{2}-m_{1}+F^{1 / 2}\left(r m_{1}-m_{2}\right)\right) / \sigma \\
& b_{2}=\left(r m_{2}-m_{1}-F^{1 / 2}\left(r m_{1}-m_{2}\right)\right) / \sigma
\end{aligned}
$$

which reduces to the $F$-distribution with one and one degree of freedom when $\mathrm{r}=\mathrm{m}_{1}=\mathrm{m}_{2}=0$.

\section{SUMMARY}

Actual uniformity yield trials are examined with respect to errors of the first and second kind, and it is found that the use of the conventional mathematical model may assess very poorly the probabilities involved. Very different fundamental error distributions were assumed, and Monte Carlo results were obtained by electronic computer methods. The $F$-distributions were somewhat robust under these models. The mathematical model was then changed to permit fertility levels to vary from subplot to subplot and also variability to vary from subplot to subplot. With such a mathematical model, it is possible to get greatly distorted $F$-distributions which exhibit many of the characteristics of actual field trials.

In general, it appears that actual field trials may grossly over- or underestimate the probabilities of errors of the first kind and the same is true for errors of the second kind. When errors of the first kind are less probable than expected, then the probability of errors of the second kind is greatly increased.

\section{ACKNOWLEDGMENTS}

The authors wish to express their gratitude to Mrs. Frances Rye Jones, who made many of the calculations in connection with the actual trials and to Miss Carol J. Lewis, who programmed the Monte Carlo machine runs. 


\section{LITERATURE CITED}

BAKER, G. A.

1932. Distribution of the means divided by the standard deviations of samples from nonhomogeneous populations. Ann. of Math. Stat. 3:1-9.

1941. Fundamental distribution of errors for agricultural field trials. Nat'l. Math. Mag. 16: $1-13$.

1952. Uniformity field trials when differences in fertility levels of subplots are not included in experimental error. Ann. of Math. Stat. $23: 289-93$.

1958. Empiric investigation of a test of homogeneity for populations composed of normal distributions. Jour. of Amer. Stat. Ass'n. 53:551-57.

BAKER, G. A. and R. E. BAKER

1953. Strawberry uniformity yield trials. Biometrics 9:412-21.

BAKER, G. A. and F. N BRIGGS

1950. Yield trials with backeross derived lines of wheat. Ann. of Inst. of Stat. Math. 2:61-67.

BAKER, G. A., M. R. HUBERTY and F. J. VEIHMEYER

1952. A uniformity trial on unirrigated barley of ten years' duration. Agron. Jour. 44:267-70.

BAKER, G. A. and E. B. ROESSLER

1957. Implications of a uniformity trial with small plots of wheat. Hilgardia $27: 183-88$.

HANNA, G. C. and G. A. BAKER

1949. Transformation of split-plot yield trial data to improve analysis of variance. Proc. of Amer. Soc. for Hort. Sci. 53:273-75.

Hoyle, B. J. and G. A. BAKeR

1961. Stability of variety response to extensive variations of environment and field plot designs. Hilgardia 30:365-94E.

RIDDLE, O. C. and G. A. BAKER

1944. Biases encountered in large-scale yield tests. Hilgardia 16:1-14. 

The journal HILGARDIA is published at irregular intervals, in volumes of about 650 to 700 pages. The number of issues per volume varies.

Single copies of any issue may be obtained free, as long as the supply lasts; please request by volume and issue number from:

\author{
Agricultural Publications \\ University Hall \\ University of California \\ Berkeley, California 94720
}

The limit to nonresidents of California is 10 separate titles. The limit to California residents is $\mathbf{2 0}$ separate titles.

The journal will be sent regularly to libraries, schools, or institutions in one of the following ways:

1. In exchange for similar published material on research.

2. As a gift to qualified repository libraries only.

3. On a subscription basis- $\$ 7.50$ a year paid in advance. All subscriptions will be started with the first number issued during a calendar year. Subscribers starting during any given year will be sent back numbers to the first of that year and will be billed for the ensuing year the following January. Make checks or money orders payable to The Regents of The University of California; send payment with order to Agricultural Publications at above address. 